\title{
MAGNETISM OF URANIUM INTERMETALLICS
}

\author{
W. SUSKI \\ W. Trzebiatowski Institute of Low Temperature and Structure Research \\ Polish Academy of Sciences \\ P.O. Box 937, 50-950 Wrocław 2, Poland \\ and \\ International Laboratory of High Magnetic Fields and Low Temperatures \\ Gajowicka 95, 53-529 Wrocław, Poland
}

In the present review first the magnetic properties of the actinide metals are presented with a special emphasis on the uranium. Then the reason for starting the research on the uranium compounds in Wroclaw is given together with a brief description of the magnetic properties of the binary compounds. The comparison with the rare-earth ternaries illustrates the difficulties in interpretation of the physical measurements of the uranium intermetallics in which the uranium as well as the transition metal atoms contribute to the magnetic ordering. Further, we present the features which could indicate the true contribution of both partners. However, we also stress that some of the properties which seem to prove the magnetic order on the uranium atom could result from particular crystal or band structure.

PACS numbers: $75.30 . \mathrm{Cr}, 75.30 . \mathrm{Gw}, 75.50 . \mathrm{Cc}, 76.80 .+\mathrm{y}$

Actinide metals form the last known family in the periodic table of the elements. Majority of them have been obtained artificially in the nuclear reactions. Although their identity results from the development of the $5 f$ shell, the similarity to other $f$ electron family (lanthanides) is limited to the heavier representatives of the $5 f$ group. The lighter actinides are very much like anomalous lanthanides, e.g. Ce, and particularly uranium and its compounds under some circumstances exhibit some similarity to the transition elements.

In Fig. 1 a schematic representation of the magnetic properties of the actinide metals is shown [1]. One can see that the magnetic ordering is observed only for heavier actinides beginning from curium. Some indication of localized magnetic moment can be detected for americium and these behaviors suggest a similarity of these elements to lanthanides.

Now, the peculiarities of physical properties of the uranium metal will be briefly discussed, because this element is a component of the compounds which are the subject of the present review. At low temperature, numerous physical properties of uranium exhibit puzzling features. At $43 \mathrm{~K}$ there is sudden expansion of the crystallographic orthorhombic cell, and the elastic constants show anomalous behavior. The similar anomalies are also observed at 23 and $37 \mathrm{~K}$ [2], which are 


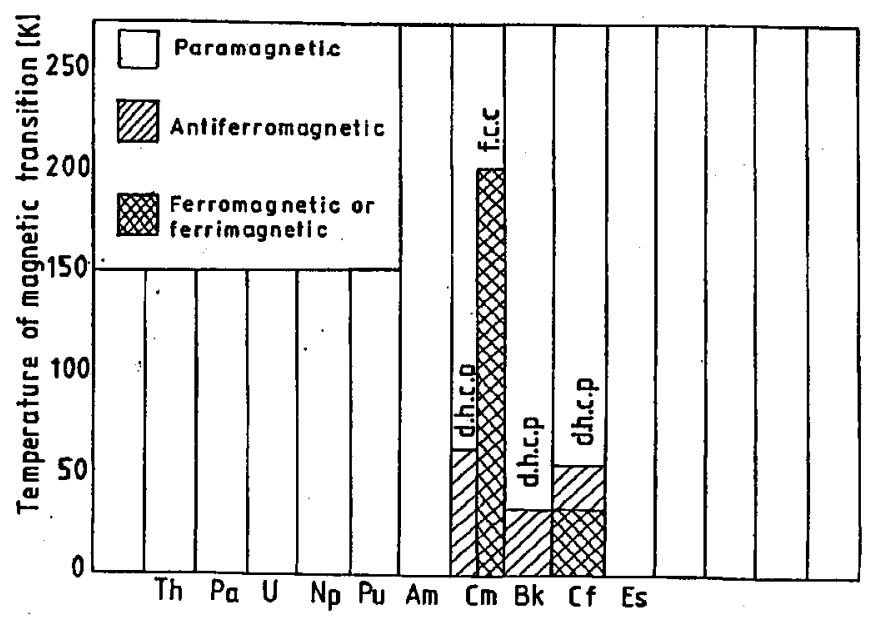

Fig. 1. A schematic representation of the magnetic order in actinide metals, reprinted from [1] with kind permission of Elsevier Science (copyright 1983).

accompanied by jumps in the lattice parameters [3], which point out to an existence of two fine isostructural phase transitions in the frame of the orthorhombic structure. These phase transitions are associated with some anomalics in the low temperature specific heat [4] and in the electrical properties [5]. As one can see from Fig. 1, the magnetic phenomena are not the reason for this beliavior, however, undoubtlessly the so-called "internal distortion" of $\alpha$-uranium is the reason for the anomalies at $43 \mathrm{~K}[6]$. This distortion was detected in careful examination on the single crystal sample. It is assumed that due to the internal distortion the condensation of electrons into almost sinusoidal wave of charge density occurs, which is incommensurate with the lattice, as it is observed in the low-dimensional compounds (e.g. $\mathrm{NbSe}_{3}$ below Peierls transition). Theoretical considerations [7] suggest that the temperatures of the anomalies correspond to the different directions of incommensurability and the phase transition at $23 \mathrm{~K}$ is to a purely commensurate phase. It is to be mentioned that similar effect as that at the temperature of $43 \mathrm{~K}$ is accomplished by pressure, which additionally induces the transition to the superconducting state [8]. However, at present we are not going to discuss this problem.

It seems that the peculiar electronic structure of uranium is the reason for those and some other anomalies. As mentioned above, the uranium behavior is different from its lanthanide analogs ( $\mathrm{Pr}$ or $\mathrm{Nd}$ ). The best explanation of the differences between electronic structures of uranium and e.g. gadolinium is provided in the schematic pictures in Fig. 2 due to Freeman [9]. In Gd the highly localized $4 f$ shell lies above the $\mathrm{Xe}$ core electron states by $\approx 10 \mathrm{eV}$ and $\approx 15 \mathrm{eV}$ below the $5 d-6 s$ valence levels, well below the Fermi energy. For uranium, there are three electronic configurations with different occupation numbers of $5 f$ shell possible which lie fairly close in energy to the $6 d-7 s$ electrons and the Fermi level. Therefore, one expects the $5 f$ electrons to contribute to the conduction processes along 


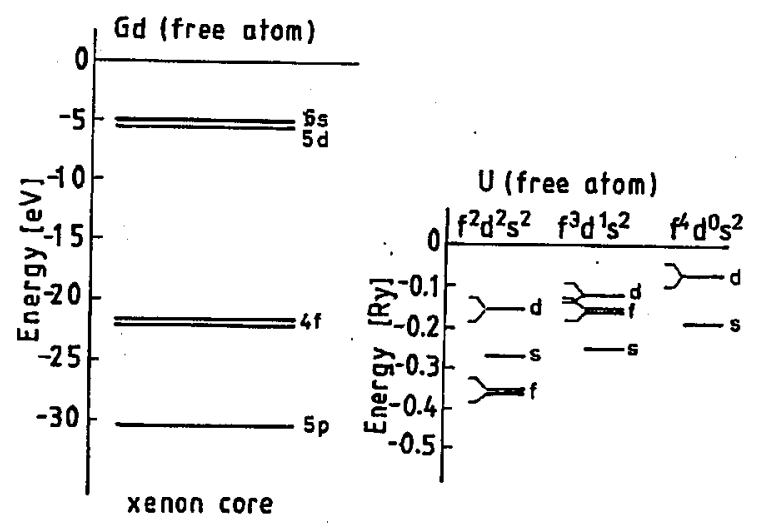

Fig. 2. The electronic structure of the gadolinium (left part) and uranium (right part) metals, reprinted from [9] with kind permission of Elsevier Science (copyright 1980).

with the $6 d$ and $7 s$ electrons and to hybridize with them strongly. Moreover, the $5 f$ electron shell being expanded in space is extremely sensitive to any influence of the external factors like pressure, magnetic and crystal fields etc. Thus, there exists a delicate balance between different types of the interactions in the uranium ion resulting in unusual properties as mentioned anomalies observed in the uranium metal but also complex magnetic ordering, spin fluctuation, heavy-fermion, Kondo lattice etc., when the uranium atom forms the compounds.

Below there are given some general remarks concerning the uranium compounds. Contrary to the uranium metal many of its compounds exhibit magnetic ordering and many other phenomena suggesting some degree of localization of the $5 f$ electrons. Most probably the main reason for this behavior is an increase in the uranium-uranium separation in compounds as compared to the pure metal $[10,11]$. In $1970 \mathrm{Hill}[12]$ observed that arranging the uranium compounds according to the $\mathrm{U}-\mathrm{U}$ distance one obtains two distinct groups of compounds. Those in which the $\mathrm{U}-\mathrm{U}$ separation is larger than critical value exhibit a magnetic ordering whereas for those in which the separation is smaller the Pauli type paramagnetism and sometimes superconductivity are observed. This value is supposed to be 3.2-3.4 $\AA$. The so-called Hill plot being phenomenological one neglects totally electronic character of components and it is one of the weaknesses of this plot. The overestimation of the importance of the $\mathrm{U}-\mathrm{U}$ separation has also led to simplification because the observed maximum in the temperature of magnetic ordering of the monocompounds inclined Grunzweig-Genossar et al. [13] to claim that the magnetic interactions in these compounds have an oscillatory character, of the RKKY-type and the compounds are analogs of the lanthanides.

Strong development of the research on the solid compounds of uranium in Poland and particularly in Wrocław was not related with any nuclear energy research establishment but resulted from discovery by Trzebiatowski and his co-workers [14] of the ferromagnetism in the uranium hydride and deuteride (for details see [15]). This discovery was followed by obtaining, frequently for the 
first time, many uranium semi- and intermetallic compounds, majority of which proved to be magnetically ordered. These binary or pseudobinary compounds exhibit practically all types of magnetic ordering and extensive review can be found in [10]. The most important conclusions from this first period of research are that the magnetic ordering in majority of these compounds occurs at temperatures below 100-200 K which are an order of magnitude higher than in corresponding compounds of lanthanides and that the uranium compounds exhibit very high magnetocrystalline anisotropy also higher than in lanthanide compounds. It should be noted that at this early period the magnetic properties of the binary compounds, later known as the heavy-fermion systems, were examined in Wroclaw [10]. Unfortunately, transport and thermodynamic properties of these materials were not checked in this distant past.

At present, the research on the uranium (and other actinides) compounds is concentrated on two main topics: a search for new heavy-fermion systems and the attempts to explain their still puzzling properties, and determination of the structure and properties of the ternary and pseudoternary compounds. Heavy-fermion systems keep to attract more vivid attention because more exciting physics seems to be engaged in these problems. The second topic mostly concerns technology of new materials and their characteristics. It does not mean that the binary compounds which are not heavy fermions, are totally neglected. The development of preparation methods results in many single crystal materials, thin films and recently fine powders accessible for the investigation of the magnetic properties. Also more advanced experimental methods provide an important information as e.g. the resonant magnetic X-ray scattering (for a review see e.g. [16]) which gives a chance for the determination of the orbital and spin part of magnetic moment.

In the present review we are going to present magnetic and related properties of some ternary and pseudoternary uranium compounds in which also transition elements are present, particularly those transition elements which exhibit magnetic ordering, namely $\mathrm{Fe}, \mathrm{Co}, \mathrm{Ni}$ and to the less extent $\mathrm{Cr}$ and $\mathrm{Mn}$. The magnetic properties of the similar compounds of the lanthanides are described by clear-cut rules. In the case of light elements their total magnetic moment is a difference between orbital and spin part. This total moment is coupled parallel to the moment of transition metal whereas for heavier lanthanides the total moment of the element is a sum of both parts but is coupled antiparallel to the moment of the transition metal. The coupling is realized through the orbital part. But these simple rules can be violated due to e.g. crystal field (CEF) interactions (see e.g. [17]) and the polarization of conduction electrons can have the similar influence. For the ternaries, which exhibit for example the $\mathrm{ThMn}_{12}$-type of structure, this scheme is experimentally confirmed when the concentration of iron-group element is high [18-20]. However, in $\mathrm{GdMn}_{12}$ [21] the mutual orientation of magnetic moments of $\mathrm{Gd}$ and $\mathrm{Mn}$ is complex. The Gd moment along the $\langle 100\rangle$ direction is parallel to the moments of the Mn atom located in part on the $8(\mathrm{j})$ positions and antiparallel to the moment of the $\mathrm{Mn}$ atoms located in part on the 8(i) positions. The fraction of the $\mathrm{Mn}$ atoms located on the residual 8(i) and 8(j) sites exhibit magnetic moments perpendicular to that of the Gd atoms and mutually antiparallel. The $\mathrm{Mn}$ atoms located on the $8(f)$ positions have very small magnetic moment. 
As far as the uranium compounds with the transition elements are concerned, the mechanism of coupling between both components is obscure and the information corresponding to the orbital part of the uranium magnetic moment is scarce. As mentioned before, an advent of development of the resonant X-ray magnetic scattering can give some light on this problem. The serious complication in actinide compounds (light) is the ratio between the $5 f$ band width and the spin-orbit coupling. For the $3 d-5 d$ elements the spin-orbit coupling is relatively weak and it can be broken by the CEF interactions, and the total spin number is a good quantum number. In turn, for the lanthanides the spin-orbit coupling is relatively strong and $J$ is a good quantum number. For the actinides, however, both interactions are comparable and therefore the $L-S$ coupling can be partially bro$k \mathrm{cn}$. Then the intermediate coupling $(j-j)$ can exist. In Table there are collected

TABLE

Magnetic properties of the binary and ternary uranium and iron compounds.

\begin{tabular}{|c|c|c|c|c|c|}
\hline Compound & $\begin{array}{l}\text { Cryst. } \\
\text { struct. }\end{array}$ & $\begin{array}{l}\text { Magn. } \\
\text { order }\end{array}$ & $\begin{array}{c}T_{\mathrm{C}, \mathrm{N}, \mathrm{S}, \mathrm{f}} \\
{[\mathrm{K}]}\end{array}$ & $\begin{array}{c}\mu_{\mathrm{s}, 0} \\
{\left[\mu_{\mathrm{B}}\right]}\end{array}$ & Ref. \\
\hline \multirow[t]{2}{*}{$\mathrm{UFe}_{2}, \mathrm{sc}$} & cubic & $F$ & $170(5)$ & $\begin{array}{l}\mu_{\mathrm{L}}=1.21(3) \\
\mu_{\mathrm{S}}=0.96(\text { at } 2 \mathrm{~T})\end{array}$ & 22 \\
\hline & $\mathrm{LavPh}$ & $\mathbf{F}$ & 162 & $\begin{array}{l}\mu_{0}=1.09 \\
\mu_{\mathrm{U}}=0.06 / \mathrm{U} \\
\mu_{\mathrm{Fe}}=0.59 / \mathrm{Fe}\end{array}$ & 23 \\
\hline $\mathrm{U}_{x} \mathrm{Fe}_{100-x}$ & LavPh & $\mathbf{F}$ & $142-172$ & & 24 \\
\hline $\mathrm{U}_{1-x} \mathrm{Ce}_{x} \mathrm{Fe}_{2}$ & $\mathrm{LavPh}$ & SG & $170-235$ & & 25 \\
\hline $\mathrm{U}_{1-x} \mathrm{Ce}_{x} \mathrm{Fe}_{2-y} \mathrm{Al}_{y}$ & $\mathrm{LavPh}$ & SG & $150-220$ & & 25 \\
\hline $\mathrm{U}\left(\mathrm{Fe}_{1-x} \mathrm{Al}_{x}\right)_{x}$ & $\mathrm{LavPh}$ & $\mathbf{F}$ & $50-170$ & & 25 \\
\hline $\mathrm{UFe}_{4} \mathrm{P}_{12}$ & cubic & $\mathbf{F}$ & 3.15 & $\mu_{\mathrm{s}}=1.0 / \mathrm{U}$ & 26 \\
\hline $\mathrm{UFe}_{2} \mathrm{Si}_{2}$ & tetr. & TIP & & & 27 \\
\hline $\mathrm{UFe}_{2} \mathrm{Ge}_{2}, \mathrm{sc}$ & tetr. & TIP & & & 28 \\
\hline $\mathrm{U}_{6} \mathrm{Fe}$ & tetr. & $\mathrm{SC}$ & 3.764 & $\gamma=157.7^{a}$ & 29 \\
\hline $\mathrm{UFeGa}_{5}$ & tetr. & WP & & & 30 \\
\hline $\mathrm{U}_{x} \mathrm{Fe}_{1-x}$ & th.a.f. & $\mathrm{CF}$ & $32^{b}$ & & 31 \\
\hline
\end{tabular}

magnetic data on the uranium compounds with iron. In all these compounds the magnetic order was detected on one component (uranium or iron) only. The reason that the magnetic order exists only in the transition metal sublattice could be almost a total compensation of the orbital and spin part of magnetic moment as 
it is seen in $\mathrm{UFe}_{2}$ ([23] and Table). The same compensation was detected also in $\mathrm{UNi}_{2}$ [32]. In turn, the magnetic order in the uranium sublattice can be easily detected when complete filling of the transition metal $d$ band occurs. The uranium ternaries in which there are some indications of magnetic moment on both the uranium and the transition metal are discussed in the following part. The most direct detection of the magnetic order can be obtained from the neutron diffraction (ND) experiment. However, in selected cases, due to various reasons this experiment is nonconclusive and therefore other indirect methods should be applied. Hence, for the compounds containing the Fe atom the Fe Mössbauer effect (ME) can provide the value of the magnetic moment for this element. Then comparison of the magnetic moment of the iron sublattice with that obtained in magnetization process at saturation allows to determine the uranium contribution to the total magnetism and also mutual orientation of the sublattice moments. However, this procedure is very often noneffective because in these highly anisotropic materials the saturation can be difficult to reach, even in applied high magnetic fields (up to $20 \mathrm{~T}$ in Wrocław) and the measurements on powder samples are not yet very popular also because of high sensitivity of such samples to air and moisture.

The other difficulty is posed by the conversion factor between ME hyperfine field and magnetic moment which is a phenomenological constant and the values from various laboratories differ considerably. The results concerning the $\mathrm{U}_{2} \mathrm{Fe}_{17-x}(\mathrm{Si}, \mathrm{Ge})_{x}$ alloys present the best illustration of this difficulty. They are derivatives of hypothetical $\mathrm{U}_{2} \mathrm{Fe}_{17}$ compound and its lanthanide analogs have some importance as hard magnetic materials. Unfortunately, the experiments of Berlureau et al. [33] have shown that only addition of $\mathrm{Si}$ and Ge stabilizes this structure. However, in single phase samples with $2 \leq x \leq 3$ the considerable amount of $\alpha-\mathrm{Fe}$, although nondetectable by X-ray diffraction (XRD), is always present. In turn, in the alloys in which $\alpha$-Fe is absent $(3.5 \leq x \leq 4.0)$ the traces of parasitic phase are present [34]. Therefore, this material is very difficult also from technological point of view. Even though saturation is easily obtained, the $10 \%$ difference between the results of $\mathrm{Fe} \mathrm{ME}$ and saturation magnetization does not provide enough evidence for estimation of the uranium contribution to the magnetic ordering. Thus, it can be seen that $\mathrm{ME}$ is very helpful but not decisive in determination of the uranium contribution to magnetic order for these type of compounds. At this point it should be noted that also comparison with the compounds containing Th; La or $\mathrm{Y}$ instead of $\mathrm{U}$ could be helpful, but for some reasons those compounds do not exist or their properties are so different, most probably because of different atomic radius and only rarely can be used as the reference materials.

Now, we are going to present the problem of possible contribution of uranium to the magnetism and its interaction with the $3 d$ metals in the framework of the $\mathrm{ThMn}_{12}$-type compounds. The interpretation of the experimental observation is difficult because in this type of structure the transition metal atoms can enter three nonequivalent crystallographic positions (see Fig. 3). The extensive review of magnetic and related properties of these materials has been published recently by the present author [35]. At least in some of these uranium ternaries ND experiments established magnetic order in the uranium sublattice and the transition metal 


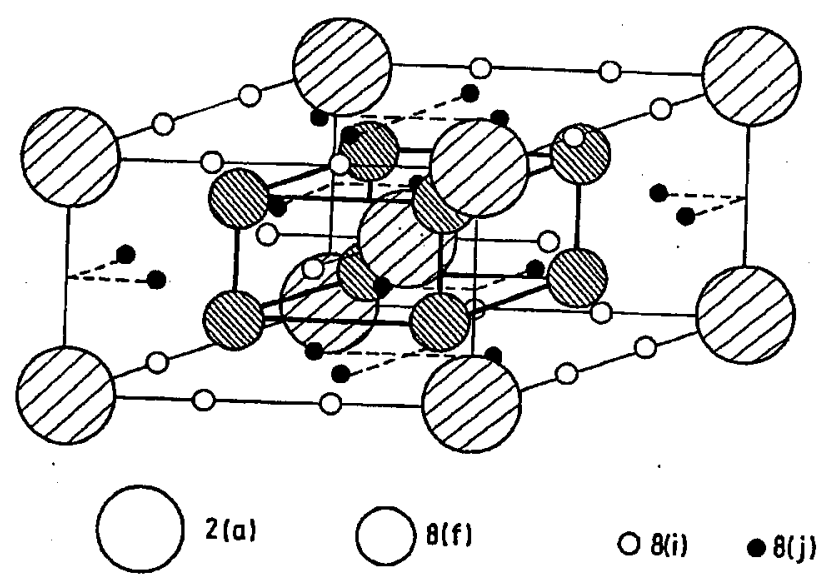

Fig. 3. The ThMnne-type of structure.

sublattice. This conclusion makes this group of compounds unique among the uranium intermetallics. Usually the uranium sublattice orders ferromagnetically, however, the magnetic moments located in various transition metal sublattices can present both ferro- or antiferromagnetic (AF) arrangements. The type of magnetic order in the sublattices can depend strongly on the separation of this last element close to the critical value for the AF interactions in the spirit of the Bethe-Slater curve. Except for this direct evidence of magnetic order in the uranium sublattice there are some experimental facts which provide also an indirect evidence.

As mentioned above, this indirect evidence can be obtained from the comparison of the results of $\mathrm{ME}$ (in case of the Fe containing compounds) and magnetometric measurements in the high magnetic field or the data for the uranium compounds and the analogs with nonmagnetic components. However, the following example can prove how doubtful can be the last procedure. $\mathrm{UNi}_{10} \mathrm{Si}_{2}$, contrary to its ferromagnetic analogs is paramagnetic [36] with a strong temperature independent susceptibility [37]. However, the lanthanide analogs exhibit magnetic ordering at low temperature. Usually, the magnetic ordering of the lanthanide compounds exist at temperature of one order lower than in the uranium compounds. Here there is a different situation. It seems to be proved that the band electrons of the rare-earth atom fill up the $\mathrm{Ni}$ band, forming a nonmagnetic state in the sublattice of this element, and at low temperature the lanthanide $4 f$ electrons are responsible for magnetic order. On the contrary, in the uranium compound the $5 f$ electrons, due to hybridization with the conduction electrons, fill up the $\mathrm{Ni}$ band and consequently both the $\mathrm{Ni}$ and $\mathrm{U}$ sublattices appear to be nonmagnetic. Therefore, this compound cannot be the reference for the other uranium compounds. $\mathrm{ScFe}_{10} \mathrm{Si}_{2}$ seems to be other reference partner with nonmagnetic Sc instead of U. The magnetic moment determined from magnetization and ME should be fairly close. However, this is not the case and the second value is twice as big as the first one. Firmly established reason for that is not known [38]. Also other experiments can be used as the evidence of the uranium magnetism. A strong anisotropic 
magnetoresistance effect in $\mathrm{UFe}_{4} \mathrm{Al}_{8}$ single crystals has been used to confirm the ferromagnetic alignment of the $\mathrm{U}$ moments, a result which would be hardly obtained by neutron diffraction experiment [39].

From those experiments it follows that in the uranium compounds the magnetic moment and the Curie/Neel point are apparently different in relation to the corresponding compounds of Sc, Y, La, Lu and Th remembering all above mentioned reservations. For some compounds the ND scattering provided sound evidence of the uranium contribution to the magnetic moment. It concerns the following compounds: $\mathrm{UCu}_{4+x} \mathrm{Al}_{8-x}$ system [40], in which the $\mathrm{AF}$ order is detected only in the $\mathrm{U}$ sublattice, $\mathrm{UFe}_{4} \mathrm{Al}_{4+x}$ [41], for which the experiments performed on single crystal sample showed AF +-+- type arrangements of the Fe moments propagating along the $a$ and $b$ axes with a ferromagnetic component on the $\mathrm{U}$ sublattice and also $\mathrm{UFe}_{5} \mathrm{Al}_{7}$ [42] in which the ferromagnetic coupling between magnetic moment of iron and uranium moments is observed. It can be seen that all ND results were obtained for the compounds with relatively low concentration of the transition element whereas for those containing more atoms of the transition element an interpretation of the ND results is very difficult.

The difference in anisotropy type between $\mathrm{UCo}_{10} \mathrm{Si}_{2}$ and $\mathrm{YCo}_{10} \mathrm{Si}_{2}$ is other argument found for $\mathrm{U}$ contribution to the magnetism. The uniaxial anisotropy of $\mathrm{UCo}_{10} \mathrm{Si}_{2}$ is relatively large; $K_{1}$ (first order anisotropy constant) exceeds its a vailable values for $\mathrm{YT}_{12-x} \mathrm{M}_{x}$ from single-crystal magnetization curves or a single-point detection method on polycrystals by a factor of 2-3. Unfortunately, there are no quantitative data on $K_{1}$ for $\mathrm{YCo}_{10} \mathrm{Si}_{2}$. However, the cone-type anisotropy found in this compound points to a low absolute $K_{1}$ value, because in the cone range a ratio $\left|K_{1}\right| \leq 2 K_{2}$ should be fulfilled, and the second anisotropy constant $K_{2}$ has never been found to be noticeable in Y-T intermetallics. Moreover, $K_{1}$ is negative in the multiaxial case. In the isostructural analogue $\mathrm{YCo}_{11} \mathrm{Ti}$ a basal plane anisotropy with $K \approx-0.9 \mathrm{MJ} \mathrm{m}^{3}$ has been found [43]. Owing to a lower Co concentration in $\mathrm{YCo}_{10} \mathrm{Si}_{2}$, the absolute value of $K_{1}$ is expected to be smaller, in agreement with observed cone-type anisotropy. Therefore, the large positive value of $K_{1}=3.3 \mathrm{MJ} \mathrm{m}$ found in $\mathrm{UCo}_{10} \mathrm{Si}_{2}$ [44] might be attributed completely to the $\mathrm{U}$ sublattice. Practically the same $K_{1}=3.0 \mathrm{MJ} \mathrm{m}^{3}$ has been observed in $\mathrm{UFe}_{10} \mathrm{Si}_{2}$ [45], which is supposed to be due to a considerable uniaxial contribution of the $\mathrm{Fe}$

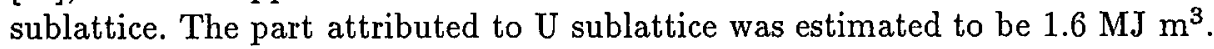

These observations, however, can result from other reasons than the $U$ contribution to magnetism. In the case of $\mathrm{Fe}$ all the $\mathrm{YFe}_{12-x} \mathrm{M}_{x}$ alloys have a uniaxial anisotropy magnitude slightly dependent on the nonmagnetic component which stabilize structure. Studies of solid solutions $\mathrm{YFe}_{12-x-y} \mathrm{Co}_{y} \mathrm{M}_{x}$ have shown that uniaxial anisotropy decreases with increasing Co content; this means that Co actually has a negative contribution to the anisotropy (see e.g. [46]). However, contrary to the results of [43], the uniaxial anisotropy has been observed in $\mathrm{YCo}_{11} \mathrm{Ti}$ [47]. The X-ray patterns of aligned powders presented in [47] show uniaxial anisotropy without any doubt. For an explanation of such a contradiction, it can be assumed that $\mathrm{YCo}_{11} \mathrm{Ti}$ has a certain homogeneity range and $K_{1}$ changes its sign within this range. The sensitivity of magnetic properties and, in particular, magnetic anisotropy to the transition metal concentration might be understood by consid- 
ering the presence of the three nonequivalent positions for other than the $f$ electron elements in the $\mathrm{ThMn}_{12}$-type lattice with different local properties. In the case of competitive anisotropy, a slight change in distribution of transition metal atoms over the nonequivalent sites can lead to a considerable change in total anisotropy. Therefore, the difference in anisotropy type between $\mathrm{UCo}_{10} \mathrm{Si}_{2}$ and $\mathrm{YCo}_{10} \mathrm{Si}_{2}$ could be attributed to different Co-Si occupancy of the positions, but not to the $U$ contribution. The same difference in the occupancy can be the reason for a different magnetic order in the transition metal sublattices for $\mathrm{UCo}_{10} \mathrm{Si}_{2}$ and $\mathrm{YCo}_{10} \mathrm{Si}_{2}$ as it was suggested for $\mathrm{ScFe}_{10} \mathrm{Si}_{2}$ [38]. The seriou's difficulty is also a small expected magnetic moment of the uranium sublattice on the high ferromagnetic background and thus all these indications are not convincing.

Finally, electronic band structure can be the reason for the differences between the uranium compounds and the compounds of their nonmagnetic ( $\mathrm{Sc}, \mathrm{Y}$, $\mathrm{La}, \mathrm{Lu}$ or Th) analogs. Particularly, the rare earth analogs could be doubtful reference materials because these last elements are trivalent as a rule, whereas uranium exhibits both the 3 - and 4 -valency. Therefore, tetravalent uranium can alter strongly the band structure in relation to the rare earth reference compounds.

It follows from the above presented review that the uranium compounds represent plenty of various physical problems. The results of the interactions of the $5 f$ electrons with the band states were discussed frequently, however, without a final conclusion. The ternaries in which only one component exhibits a magnetic order were discussed in the past $[10,11]$. However, the ternaries with two magnetic components are discussed so extensively for the first time. It was also an intention of the author to show the number of problems which are waiting for solution. Particularly, these problems should represent a challenge for the fellow-theoreticians, because only preliminary work in this field has been done [48].

\section{References}

[1] P.G. Hurray, S.E. Nave, R.G. Haire, J. Less-Common Met. 93, 293 (1983).

[2] E.S. Fisher, D. Dever, Phys. Rev. 170, 607 (1968).

[3] M.O. Steinitz, C.E. Burleson, J.A. Marcus, J. Appl. Phys. 41, 5057 (1970); D.A. Tindall, M.O. Steinitz, J. Phys. F, Met. Phys. 14, 1131 (1984).

[4] J. Crangle, J. Temporal, J. Phys. F, Met. Phys. 3, 1097 (1973).

[5] M.B. Brodsky, N.J. Griffin, M.D. Odie, J. Appl. Phys. 40, 895 (1969).

[6] H.G. Smith, G.H. Lander, Phys. Rev. B 30, 5407 (1984).

[7] M.B. Walker, Phys. Rev. B 34, 6830 (1986).

[8] T.F. Smith, E.S. Fisher, J. Low Temp. Phys. 12, 631 (1973).

[9] A.J. Freeman, Physica B 102, 3 (1980).

[10] J.M. Fournier, R. Troć, in: Handbook on the Physics and Chemistry of the Actinides, Vol. 2, Eds. A.J. Freeman, G.H. Lander, Elsevier, New York 1985, p. 29.

[11] V. Sechovsky, L. Havela, in: Handbook of Magnetic Materials, Vol. 4, Eds. E.P. Wohlfarth, K.H.J. Buschow, Elsevier, Amsterdam 1988, p. 310.

[12] H.H. Hill, in: Plutonium 1970 and Other Actinides, Ed. W.N. Miner, AIME, New York 1970, p. 2.

[13] J. Grunzweig-Genossar, M. Kuznietz, F. Friedman, Phys. Rev. 173, 562 (1968). 
[14] W. Trzebiatowski, A. Śliwa, B. Staliński, Roczn. Chem. 26, 110 (1952); ibid. 28, 12 (1954).

[15] R. Troć, W. Suski, J. Alloys Comp. 219, 1 (1995).

[16] G.H. Lander, W.G. Stirling, S. Langridge, D. Gibbs, J. Magn. Magn. Mater. 140-144, 1349 (1995).

[17] W.E. Wallace, Prog. Solid State Chem. 16, 127 (1986).

[18] W.G. Haije, J. Spijkerman, F.R. de Boer, V. Bakker, K.H.J. Buschow, J. Less-Common Met. 162, 283 (1990).

[19] R.B. Helmholdt, J.J.M. Vleggan, K.H.J. Buschow, J. Less-Common Met. 138, L11 (1988).

[20] J. Hu, T. Wang, S.G. Zhang, Y.Z. Wang, Z.X. Wang, J. Magn. Magn. Mater. 74, 22 (1988).

[21] N. Okamoto, H. Nagai, H. Yoshie, A. Tsujima, T. Hihara, J. Magn. Magn. Mater. 70, 299 (1987).

[22] B. Lebech, M. Wulff, G.H. Lander, J. Rebizant, J.C. Spirlet, A. Delapalme, J. Phys., Condens. Matter 1, 10229 (1990).

[23] A.T. Aldred, G. Cinander, D.J. Lam, L.W. Weber, Phys. Rev. B 19, 303 (1979).

[24] P.P. Vaisheva, A.E. Dwight, D. Berggren, L. Sill, Hyperfine Interact. 51, 949 (1989).

[25] S.B. Roy, G. Williams, B.R. Coles, J. Phys. (France) 50, 2773 (1989).

[26] R.P. Guertin, C. Rossel, M.S. Torikachvili, M.W. Mc Elfresh, M.B. Maple, S.H. Bloom, Y.S. Yao, M.V. Kirić, G.P. Meisner, Phys. Rev. B 36, 8865 (1987).

[27] A. Szytuła, S. Siek, J. Leciejewicz, A. Zygmunt, Z. Ban, J. Phys. Chem. Solids 49, 1119 (1988).

[28] A.J. Dirkmat, T. Enstra, E. Kuetsch, A.A. Menovsky, J.A. Mydosh, J. Phys. (France) Colloq. 49, C8-475 (1988).

[29] K.N. Yang, M.B. Maple, L.E. Delong, J.G. Hubert, A. Janod, Phys. Rev. B 39, 15 (1989).

[30] A.V. Andreev, M.I. Bartashevich, Fiz. Met. Metall. 62, 266 (1986).

[31] P.P. Freitas, T.S. Plaskett, J.M. Moreira, V.S. Amarel, J. Appl. Phys. 64, 5453 (1988).

[32] J.M. Fournier, A. Boeuf, P.H. Fringgs, M. Bonet, J.X. Boucherle, A. Delapalme, A.A. Menovsky, J. Less-Common Met. 121, 249 (1986).

[33] T. Berlureau, B. Chevalier, L. Fournes, J. Etourneau, Mater. Lett. 2, 21 (1989).

[34] J. Olejniczak, W. Suski, K. Wochowski, T. Mydlarz, in: Abstr. of 26 $6^{\text {ièmes }}$ Journées des Actinides, Szklarska Poręba (Poland) 1996, Institute of Low Temperature and Structure Research, Polish Academy of Sciences, Wroclaw, p. 91.

[35] W. Suski, in: Handbook on the Physics and Chemistry of Rare Earths, Vol. 22, Eds. K.A. Gschneidner, Jr., L. Eyring, Elsevier, Amsterdam 1996, p. 143.

[36] W. Suski, A. Zaleski, B. Badurski, L. Folcik, K. Wochowski, B. Seidel, C. Geibel, F. Steglich, J. Alloys Comp. 198, L5 (1993).

[37] W. Suski, K. Wochowski, A. Zygmunt, J. Janczak, B. Nowak, K. Niedźwiedź, O.J. Żogal, J. Alloys Comp. 199, 67 (1993).

[38] O.I. Bodak, J. Stępień-Damm, H. Drulis, B. Kotur, W. Suski, F.G. Vagizov, K. Wochowski, T. Mydlarz, Physica B 210, 183 (1995). 
[39] G. Bonfait, M. Godinho, P. Estrela, A.P. Gonçalves, M. Almeida, J.C. Spirlet, Phys. Rev. B 53, R480 (1996).

[40] A. Krimmel, A. Loidl, C. Geibel, F. Steglich, G.J. McIntyre, J. Magn. Magn. Mater. 103, 73 (1992).

[41] J.A. Paixão, A.P. Gonçalves, M. Almeida, G. Bonfait, M. Godinho, P. Burlet, in: Proc. 24 ièmes Journées des Actinides, Obergurgl (Austria) 1994, University of Wien, Wien, p. 101.

[42] K. Rećko, M. Biernacka, L. Dobrzyński, D. Satuła, J. Waliszewski, W. Suski, K. Wochowski, G. André, F. Bourée, 1996, to be published.

[43] M. Solzi, L. Pareti, O. Moze, E. David, J. Appl. Phys. 64, 5084 (1988).

[44] A.V. Andreev, M.I. Bartashevich, S.S. Sigaev, D.A. Andreev, W. Suski, K. Wochowski, T. Goto, J. Alloys Comp. 230, 30 (1995).

[45] A.V. Andreev, M.I. Bartashevich, H. Aruga Katori, T. Goto, J. Alloys Comp. 216, 221 (1994).

[46] H.S. Li, J.M.D. Coey, in: Handbook of Magnetic Materials, Vol. 6, Ed. K.H.J. Buschow, North-Holland, Amsterdam 1991, p. 1.

[47] K. Odashi, H. Ido, Y. Yoneda, J. Appl. Phys. 70, 5986 (1991).

[48] S.F. Matar, B. Chevalier, J. Etourneau, J. Magn. Magn. Mater. 137, 293 (1994). 